\title{
Coupling of orthogonal polarization states in a nonlinear birefringent cavity
}

\author{
Kwongchoi Caisy Ho and Guy Indebetouw
}

\begin{abstract}
We discuss the coupling of orthogonally polarized beams in a nonlinear cavity and show experimentally how this can be used to implement gates or latches in which one polarization state is switched by another. These devices must be reset by interrupting a light beam and thus must dissipate energy to switch down. The possibility of up and down switching with positive pulses only is also discussed.
\end{abstract}

\section{Introduction}

Polarization bistability (POB) was recently described as having a number of advantages over conventional optical bistable schemes. 1,2 These include access to complementary logic, the possibility of designing latches and gates biased by one polarization state and switched by another, and the eventual possibility of using positive pulses only for up and down switching. This last attribute is particularly interesting since it would mean that in principle down switching could be achieved without energy dissipation. ${ }^{1}$

The purpose of this paper is to comment on these advantages. We first show that a simple nonlinear birefringent cavity cannot be reset with a positive pulse but that this could be achieved in coupled cavities. We then discuss the coupling of the two polarization states in the cavity and show with some examples that this coupling can be exploited to implement certain latches and gates.

\section{Positive Pulse Only Switching}

Polarization bistability can be realized in a nonlinear birefringent Fabry-Perot cavity. The nonlinear material can itself be birefringent, or a fixed birefringent plate can be used in series with an isotropic cell. Such a cavity has two eigenpolarization states parallel $(e)$ and normal (o) to the birefringent axis. In the framework of a plane wave theory the transmittance of

The authors are with Virginia Polytechnic Institute \& State University, Physics Department, Blacksburg, Virginia 24061-0435.

Received 14 February 1989.

0003-6935/90/020206-04\$02.00/0.

(C) 1990 Optical Society of America. the cavity (ratio of transmitted to incident intensity) is proportional to an Airy function ${ }^{3}$

$$
T_{o(e)}=I_{t o(e)} / I_{i o(e)}=a_{o(e)}\left[1+F_{o(e)} \sin ^{2} \theta_{o(e)}\right]^{-1},
$$

where $a_{o(e)}$ is a function of the mirror reflectance and the absorption of the medium and $F_{o(e)}$ is the finesse of the cavity. These parameters may be different for each polarization. The phase $\theta_{o(e)}$ is the phase shift experienced by the ordinary (extraordinary) polarization after a single pass in the cavity. If the material has a nonlinearity of the Kerr type,

$$
\theta_{o(e)}=\left[n_{o(e)}+n_{2 o(e)} \eta k d+\phi_{o(e)},\right.
$$

where the first two terms are the linear and nonlinear contribution to the index of refraction, $k$ is the wavenumber in vacuum, $d$ is the cavity length, $\phi$ is an eventual phase shift due to reflection on the cavity mirrors, and $I$ is the irradiance in the cavity.

Equation (2) shows that the resonance peaks of the cavity $\left[\theta_{o(e)}=n 2 \pi\right]$ occur for different values of the phase for the two polarizations, and the rates at which these peaks shift as a function of $I$ may also be different. The two polarizations will switch independently for different values of the input and will have different hysteresis loops. ${ }^{3}$ As shown in Ref. 1, some interesting switching behavior can be obtained if the two hysteresis curves partially overlap and if the output of the device is taken as a superposition of transmitted and/ or reflected beams of each polarization. The output of the device can, for example, be taken as the sum of the reflected beam $R_{o}$ in one polarization and the transmitted beam $T_{e}$ in the orthogonal polarization as shown in Fig. 1. One can see graphically that, whatever the initial states are for $R_{o}$ and $T_{e}$, the device eventually falls into a state from which it cannot be displaced with a positive pulse. For example, the system can be biased to point $A$ in Fig. 1, where it is in the state $\left(T_{e}, R_{o}\right)=(\mathrm{LO}, \mathrm{HI})$. A weak pulse bringing the operat- 
ing point momentarily to $B$ will switch the device to the state (LO,LO). A more intense pulse bringing the system to $C$ momentarily will switch both the (LO,HI) and $(\mathrm{LO}, \mathrm{LO})$ states to a $(\mathrm{HI}, \mathrm{LO})$ state where the device is locked. It takes a negative pulse to reset it from that state. This reduces the attractiveness of polarization switching but it does not entirely abolish its other advantages.

An interesting way of resetting the device was suggested by Lohmann. ${ }^{4}$ It consists of rotating the initial polarization of the source to align it with one of the axes of the birefringent cavity. This can be achieved by using a fast Pockels cell to rotate the polarization to align it momentarily with the $o$-ray to switch the device from the locked (HI,LO) state to a $(\mathrm{LO}, \mathrm{LO})$ state. This is equivalent to interrupting the e-beam, with the important difference that the total power on the device remains constant. The system can also be switched to a (HI,HI) state by rotating the source polarization along the e-ray. To switch down it is not necessary to align the polarization completely. It needs only to be rotated by an amount sufficient to shift the cavity off resonance, which can be small for a high finesse cavity.

There are other ways of achieving positive pulse only switching. One is to use two physically different mechanisms for the up and down switching. The previous example falls into this category. Another, which we are presently investigating, makes use of two coupled cavities. In such a device one cavity can be biased by the light reflected on a second cavity. 5,6 When this auxiliary cavity is turned to a high transmission state by a positive pulse, the bias drops, turning the main cavity to a low transmission state. To implement this scheme one can use two separate cavities or a birefringent cavity addressed by two orthogonal polarizations at two separate locations.

\section{Coupling of Orthogonal Polarization States}

We now turn to the second part of the paper concerning the coupling of orthogonally polarized beams in the cavity and show experimentally how this coupling can be used to implement latches and gates.

Polarization bistability has been demonstrated in a number of systems. ${ }^{7-12}$ In all these experiments the two orthogonal polarizations are carried by the same beam and interact at the same location in the cavity. In such cases the two polarization states always switch simultaneously even if they switch independently for different values of the input. This is implicit in the analysis of Ref. 2, but for the sake of completeness we reiterate the statement using a simple argument.

From Eqs. (1) and (2) the transmittance of the cavity for each polarization is seen to be a single-valued function of the total irradiance $I=I_{0}+I_{e}$ inside the cavity. The irradiance $I_{o}$ and $I_{e}$ are proportional to the transmitted irradiance

$$
I_{o(e)}=b_{o(e)} T_{o(e)} I_{i o(e)},
$$

where $b_{o(e)}$ depends on the mirror reflection coefficient. An equation can thus be written for the total irradiance:

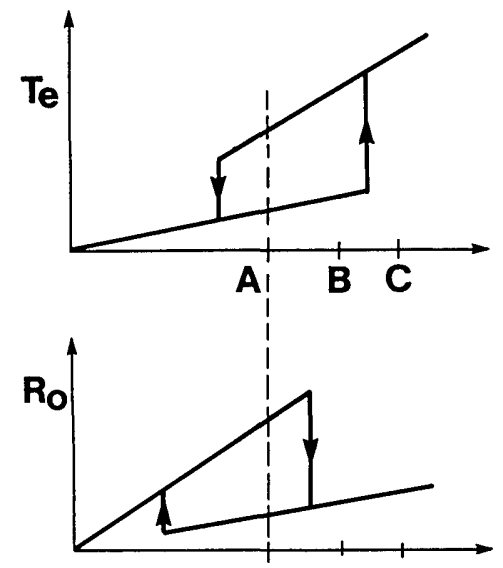

input intensity

Fig. 1. Transmission and reflection of a nonlinear birefringent cavity. The output is taken as a combination of the transmitted ebeam and the reflected $o$-beam.

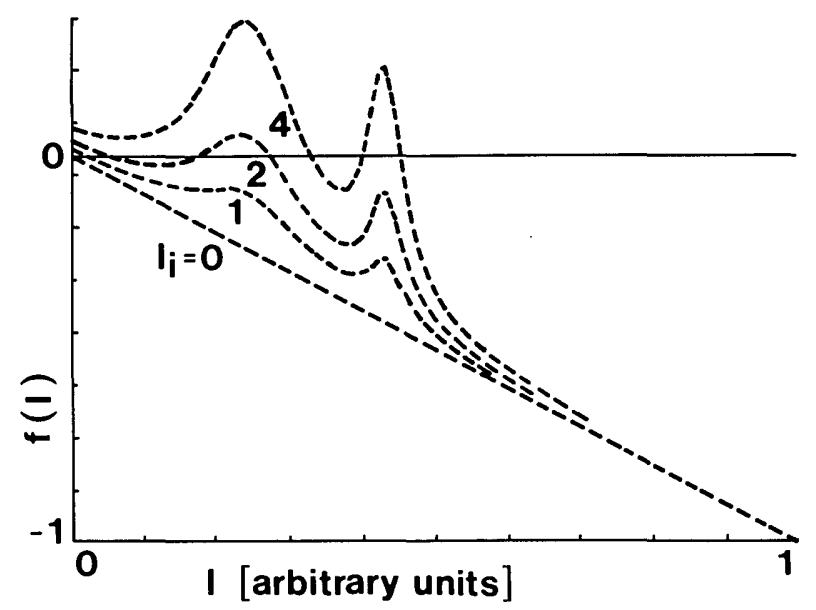

Fig. 2. Graphic representation of Eq. (4). The roots of the equation $f(I)=0$ determine the possible values of irradiance $I$ in the cavity for a given input irradiance $I_{i}$.

$$
f(I)=b_{o} T_{o}(I) I_{i o}+b_{e} T_{e}(I) I_{i e}-I .
$$

The roots of this equation determine the possible values of $I$ for a given input. Figure 2 is a graphic representation of Eq. (4), using $I_{i o}=I_{i e}=I_{i}$ and the parameter values pertaining to a cavity filled with a nematic liquid crystal (MBBA): $n_{o(e)}=1.56(1.80)$, $n_{2 e},=-3 n_{2 o}$ for thermooptic effects, $d=50 \mu \mathrm{m}$, mirror reflection coefficients $=0.85$, and medium absorption coefficient $=7.5 \mathrm{~cm}^{-1}$. For small input intensity there is only one root. Above a critical value of $I_{i}$ there are three roots for $I$; two are stable and one is unstable. This is the bistable region. For higher input intensity other roots appear as the successive peaks of the Airy function cross the axis. Clearly, if $I$ has two stable solutions for a particular input $I_{i}, T_{o, e}(I)$ also has two stable solutions. As a consequence, the two polariza- 


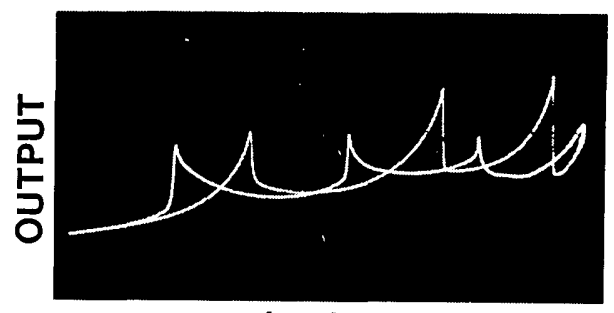

INPUT (AU)

Fig 3. Input/output characteristic curve of an MBBA filled FabryPerot cavity with metallic mirrors showing multiple switching with butterfly type hysteresis.

tion states always switch for the same critical value of $I_{i}$. The only way to avoid this indirect coupling is to spatially separate the two orthogonally polarized beams in the cavity. Some schemes to achieve this, for example, with the aid of Wollaston prisms, were suggested in Ref. 1.

\section{Experimental Results}

We have performed experiments with a Fabry-Perot cavity filled with a nematic liquid crystal (MBBA) in a planar orientation. This system takes advantage of the large thermooptic coefficient of this material. The metallic mirrors of the cavity absorb some energy, raising the temperature of the cell locally and inducing a change of refractive index. Switching occurs when this change brings the cavity closer to resonance, thus providing a positive feedback.

Two mutually parallel orthogonally polarized beams were focused in the $50-\mu \mathrm{m}$ thick cavity onto two spots $50 \mu \mathrm{m}$ in diameter. The spacing between the two spots could be varied without changing the beams' orientation. Because of transverse heat diffusion, the response of the device is not local. We found that, to achieve independent switching of the two beams, they had to be about ten beam diameters $(0.5 \mathrm{~mm})$ apart in the cell. This result is strongly dependent on the particular geometry used. This figure can be considerably reduced by pixellation of the mirrors.

The coupling of the two orthogonally polarized beams in the cavity is a drawback for certain POB devices but it can be exploited to implement various latches and gates, as demonstrated in the following experiments. The MBBA filled Fabry-Perot cavity used had metallic (Al) mirrors. The phase shift on reflection from the mirrors is responsible for the butterfly shape of the hysteretic input/output curve shown in Fig. 3. ${ }^{12}$ The lowest critical input power achieved for the first switching was below $1 \mathrm{~mW}$ and the fastest switching time was smaller than $1 \mathrm{~ms}$. Conventional hysteresis curves can be obtained by replacing one of the mirrors by a dielectric mirror. ${ }^{12}$ However, the all metallic cavity is much simpler to fabricate and is used here for the purpose of demonstration.

Figure 4(A) shows the response of a latch in which one polarization carries a clock signal $C$ to switch an orthogonally polarized signal beam $S$. The shape of the output can be explained by referring to the hyster-

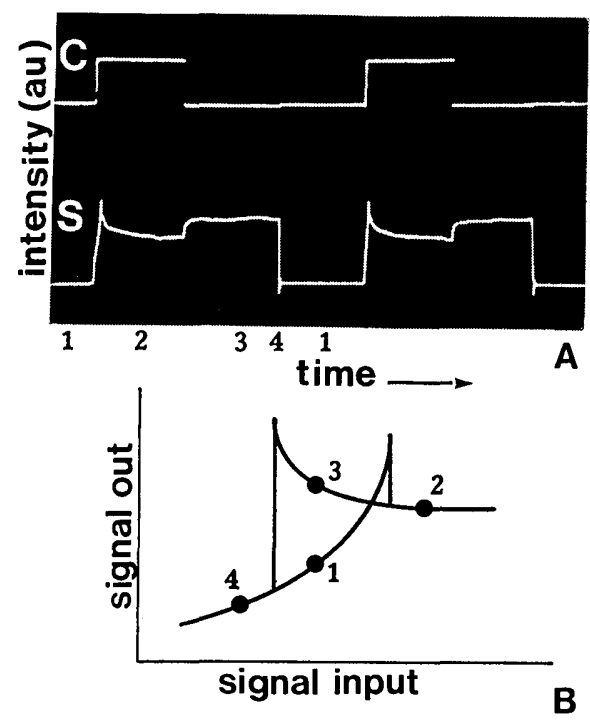

Fig. 4. Example of a latch using an orthogonally polarized clock and signal. (A) Input clock $C$ and output signal $S$ as a function of time. Reset (at point 4 ) is achieved by momentarily interrupting the signal. The signal frequency was of the order of several Hz. (B) Hysteresis curve showing the various signal levels appearing in the switching sequence of (A).

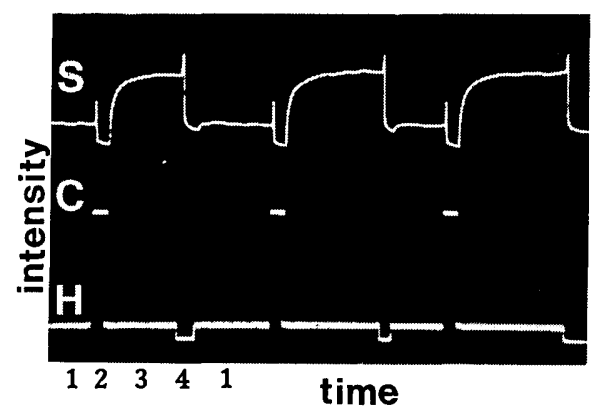

Fig. 5. Latching similar to that shown in Fig. 4 with resetting achieved by momentarily interrupting a small holding beam $H$ added to the clock.

esis curve in Fig. 4(B). Initially, the device is biased in state 1. Turning the clock oN translates the hysteresis curve to the left, shifting the transmitted signal beam from state 1 to state 2 . Interrupting the clock returns the signal to state 3 . To reset, the input signal is momentarily interrupted. This brings the output to state 4 and then immediately back to its original state 1 when the input signal is restored.

Reset can also be achieved by interrupting a constant background (holding beam) added to the clock signal. This is shown in Fig. 5. The action of clock $C$ bringing the output from state 1 to state 3 is as in the previous case. Interrupting the holding beam $H$ momentarily shifts the hysteresis curve to the right, driving the output to state 4 . When the holding signal is restored the output goes back to its original state 1 . For Fig. 5 the shape of the hysteresis curve is similar to the one shown in Fig. 4(B) except for the output level of state 2 which is lower than that of state 1.

These simple examples show how the coupling of two orthogonal polarizations in a nonlinear cavity can 
be exploited to implement latches and gates. It should be stressed, however, that these devices do not necessarily require a birefringent cell. They simply use two orthogonal polarization states to carry different information (signal, clock, or hold) independently and separably.

The constructive comments of the referees are appreciated. One of us (GI) would like to acknowledge some very fruitful discussions with A. Lohmann on the subject of this paper.

\section{References}

1. A. Korpel and A. W. Lohmann, "Polarization and Optical Bistability," Appl. Opt. 25, 1528-1529 (1986).

2. A. Korpel and A. W. Lohmann, "Criteria for Optical Bistability in Ring Cavities with Two Orthogonal Modes of Polarization," Appl. Opt. 25, 2253-2257 (1986).

3. M. M. Gibbs, Optical Bistability: Controlling Light with Light (Academic, New York, 1985).

4. A.W. Lohmann, Physikalisches Institut der Universitat Erlangen-Nurnberg; private communication.
5. A. Szöke, V. Daren, J. Goldhar, and N. A. Kurnit, "Bistable Optical Element and Its Applications," Appl. Phys. Lett. 15, 376-379 (1969).

6. K. Otsuka, "Pitchfork Bifurcation and All-Optical Digital Signal Processing with a Coupled-Element Bistable System," Opt. Lett. 14, 72-74 (1989).

7. I.P. Areshev, T. A. Murina, N. N. Rosanov, and V. K. Subashiev, "Polarization and Amplitude Optical Multistability in a Nonlinear Ring Cavity," Opt. Commun. 47, 414-419 (1983).

8. Y. C. Chen and J. M. Liu, "Polarization Bistability in Semiconductor Lasers," Appl. Phys. Lett. 46, 16-18 (1985).

9. R. Cush and C. T. G. Kirkby, "Polarization Bistability in a Nonlinear Birefringent Fabry-Perot Cavity,"Opt. Commun. 60, 399-404 (1986).

10. A. D. Lloyd, "Polarization Optical Bistability in Nematic Liquid Crystals," Opt. Commun. 64, 302-306 (1987).

11. G. Indebetouw, "Polarization Bistability in a Hybrid Device Simulating a Nonlinear Birefringent Cavity," Opt. Commun. 65, 311-314 (1988).

12. D. C. Hutchings, A. D. Lloyd, I. Janossy, and B. S. Wherrett, "Theory of Optical Bistability in Metal Mirrored Fabry-Perot Cavities Containing Thermo-Optic Materials," Opt. Commun. 61, 345-350 (1987). 